\title{
Squamous Cell Carcinoma of the Thyroid Gland in an elderly Female
}

\author{
Talal Mohammed Al-Shahri ${ }^{1 *}$, Dema Wahid Hammami ${ }^{1 \mathrm{a}}$, Mutassem Jaber ${ }^{1 \mathrm{~b}}$ and Ghadeer Mazlooh Alenezi ${ }^{1 \mathrm{c}}$ \\ ${ }^{1}$ Department of Surgery, Imam Abdulrahman Alfaisal Hospital, Riyadh, Saudi Arabia.
}

Corresponding Author: Talal Mohammed Al-Shahri, Department of Surgery, Imam Abdulrahman Alfaisal Hospital, Riyadh, Saudi Arabia.

Received Date: March 27, 2021; Accepted Date: April 07, 2021; Published Date; April 09,2021

Citation: Talal M. Al-Shahri, Dema W. Hammami, Jaber M. and Ghadeer M. Alenezi. (2021) Squamous cell carcinoma of the thyroid gland in an elderly female. J. Surgical Case Reports and Images. 4(3); DOI:10.31579/2690-1897/066

Copyright: ( 2021 Talal Mohammed Al-Shahri, This is an open-access article distributed under the terms of the Creative Commons Attribution License, which permits unrestricted use, distribution, and reproduction in any medium, provided the original author and source are credited.

\begin{abstract}
Background: Squamous cell carcinoma of the thyroid gland is a rare tumor. The tumor may arise as a primary tumor within the thyroid gland.

Case Presentation: A 62-years-old lady with history a history of a long-standing left neck mass presented with progressive enlargement of the mass for the last 2 months. The mass was associated with dyspnea, dry cough, and difficulty in swallowing but no change of voice. On examination, it was hard and fixed, and other parts of the general examination were unremarkable. Fine needle aspiration revealed malignant cells with squamous cell cancer (BETHESDA VI). On surgical exploration, there was a mixed cystic and solid mass arising from the left thyroid gland locally invading strap muscles, esophagus and trachea. Complete excision was not possible, debulking was done with modified radical neck dissection. The histopathology showed Invasive poorly differentiated squamous cell carcinoma of the thyroid with cystic changes in background of Hashimoto's thyroiditis.
\end{abstract}

Conclusion: Thyroid squamous cell carcinoma is a rare malignancy with significant management implications. Less than three hundred clear cases of thyroid squamous cell carcinoma have been reported. In this report we describe one additional case of a thyroid squamous cell carcinoma and provide a comprehensive discussion of the clinical significance, and appropriate surgical management.

Keywords: squamous cell carcinoma, thyroid cancer, neck mass, thyroid

\section{Introduction}

Primary Squamous cell carcinoma (SCC) of the thyroid gland is a rare malignancy, constituting <1\% of all thyroid malignancies [1]. Commonly presents as a neck mass with compression symptoms such as dyspnea and dysphagia. It is usually aggressive and invading surrounding organs.

The distinction between primary SCC of the thyroid and an invasion of SCC arising in the adjacent organs is important because of the poor prognosis of thyroid SCC [2]. Diagnosis is made by histopathology. Three immunochemical markers are usually detected in differentiated thyroid carcinomas, PAX8, thyroglobulin (Tg), and TTF-1. PAX8 was found to be negative in pure SCC of thyroid which is useful in distinguishing primary SCC from invasion or metastasis from extrathyroidal SCC [2].

Complete surgical resection is the recommended treatment; however, the benefit of adjuvant therapy is still unclear and does not impact overall survival if complete surgical resection is done [3].

\section{Case Presentation}

A 62-year-old lady with a history of long-standing neck swelling presented with progressive enlargement of a left neck swelling for 2 months which was associated with dyspnea and dry cough and difficulty in swallowing, No hoarseness of voice, no family history of cancer no history of radiation or smoking. The mass was hard and fixed not moving with swallowing no lymphadenopathy, no eyes signs other parts of the general examination were unremarkable.

Thyroid stimulation hormone was high $6.0 \mathrm{miu} / \mathrm{L}$, thyroxine (T4) and Triiodothyronine (T3) were normal. Other investigations like Ca level were within normal limits $2.12 \mathrm{mmol} / \mathrm{L}$, Phosphorus normal $1.2 \mathrm{mmol} / \mathrm{L}$, alkaline phosphatase within normal $124 \mathrm{U} / \mathrm{L}$, Parathyroid hormone normal $2.50 \mathrm{pmol} / \mathrm{L}$, LDH was high 264U/L.

Ultrasound neck showed: enlarged both thyroid lobes mainly on the left with multiple nodules largest on left lobe measuring $4.2 \times 3 \mathrm{~cm}$ with cystic degeneration and coarse calcifications and increased vascularity. Fine needle aspiration was done for the mass which revealed malignant cells with squamous cells BETHESDA VI, CT scan of the neck was done showing a large complex left thyroid lobe mass with large cystic/necrotic component and coarse calcification centrally, measuring $6.5 \mathrm{X} 6 \mathrm{X} 4$ $\mathrm{cm}$. The mass is causing mass effect and right sided deviation. No retrosternal extension. The left internal jugular vein is severely 
compressed by the lesion causing complete collapse and nonopacification of the vein segment at the level of the lesion. There are associated necrotic lymph nodes inferior to the mass, in keeping with nodal metastasis, the largest lymph node measures approximately $1 \mathrm{~cm}$ with a thick enhancing rim. A few similar but smaller lymph nodes are noted at the level of left III and IV. No other masses throughout the neck. No intraorbital or intracranial abnormalities. No suspicious osseous lesion.

CT scan of brain, chest, abdomen and pelvis endoscopy, colonoscopy and bronchoscopy no primary tumor or metastasis.

At surgery, a $7 \mathrm{~cm}$ complex mix cystic to solid mass was identified in the left thyroid lobe which locally invading the strap muscles, trachea, and esophagus. There were multiple hard calcified lymph nodes fixed to the tumor. En-block total thyroidectomy with modified radical neck dissection of levels $3,4,5$, and 6 was performed. The parathyroid glands and recurrent laryngeal nerve were preserved.

The histopathology of the resected specimen showed a $6 \times 6 \mathrm{~cm}$ invasive poorly differentiated squamous cell carcinoma of the thyroid with cystic changes in a background of Hashimoto's thyroiditis. There were no extrathyroidal (capsule) extension and no lymphovascular invasion, but there was a positive central lymph node metastasis, $4 / 10$. The right thyroid lobe showed Hashimoto's thyroiditis.

\section{PET scan}

A follow up post-operative PET scan showed a large heterogeneous FDG avid (SUVmax up to 13.6) ill-defined soft tissue density/mass lesions with surrounding multiple focal FDG avid nodal lesions and central photogenic fluid density with multiple air pockets seen in the vicinity of the left thyroid bed extending to the left mid-cervical region and suprasternal region); most likely related to recent postoperative changes. There is an intense FDG avid (SUVmax 7.2) right upper cervical lymph node. No other significant FDG avid lymphadenopathy is noted elsewhere in the study. There are multiple scattered moderate intense FDG avid focal hypodense hepatic lesions. There is a focal intense FDG avid (SUVmax 12.7) destructive lytic bony lesion seen involving C5 vertebral body. Besides, there is a small focal moderate FDG avid (SUVmax 6.4) cortical lytic bony lesions seen involving the right femur posterior subtrochanteric region. Furthermore, there is a small mild focal increased FDG activity (SUVmax 2.1) seen at the left iliac bone. Lungs showed a few scattered small bilateral pulmonary nodules, which are below the resolution of PET. There is atelectatic/bronchiectatic changes seen at the right middle lobe with no significant increased FDG activity. There are small non-avid pericardial and right pleural effusions. Otherwise, physiological tracer distribution is noted elsewhere in the study with no significant focal FDGavid lesions seen at the brain, spleen, adrenals, or pancreas.

IMPRESSION: The described heterogeneous intense FDG avid soft tissue density/mass lesion at the lower neck is highly suspicious for recent postoperative changes along. FDG avid metastatic lymphadenopathy in the neck, as described. Multiple FDG avid metastatic hepatic lesions. Few FDG avid metastatic bony lesions. The few scattered small bilateral pulmonary nodules are highly suspicious for metastasis. The patient received courses of radiotherapy in a tertiary center.

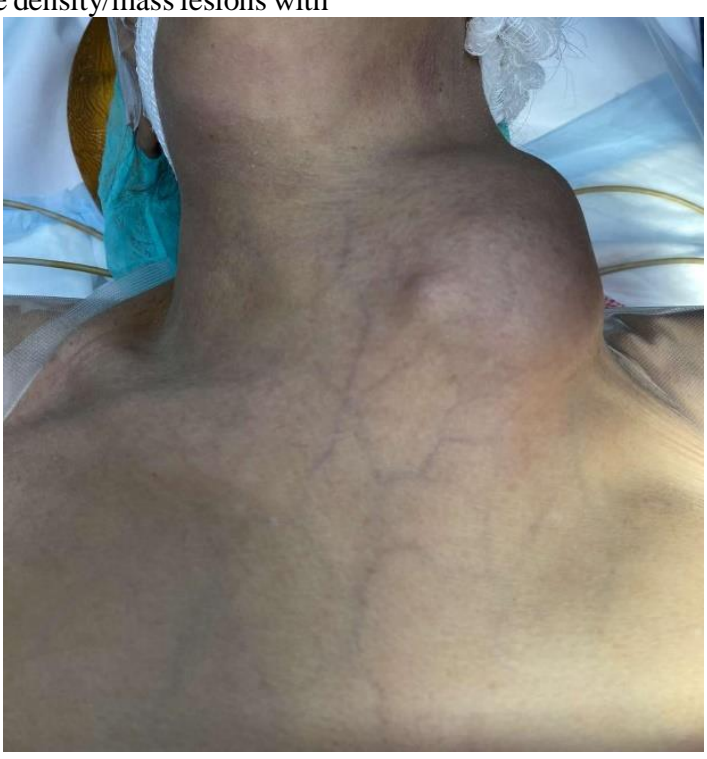

Figure1. A preoperative photograph showing the mass on the left side the neck with multiple dilated superficial veins resulting from left internal jugular vein compression

\section{Discussion}

Primary squamous cell carcinoma (PSCC) was found to affect women in their seventh decade of life, with the mean age of diagnosis at 68 years and a female: male ratio of 1.4:1 [1].

As it was reported in most previous literature, patients usually present with compression symptoms such as dyspnea and dysphagia. Our patient presented with progressive enlargement of a left neck nodule for 2 months which was associated with dyspnea and dry cough and hoarseness of voice. Hypercalcemia and leukocytosis were observed also with PSCC of the thyroid [4].
Most cases reported were poorly differentiated carcinoma as in our case. Because of lack of squamous cells in the thyroid gland the occurrence and the origin of the thyroid squamous cell carcinoma is a matter of debate. Two theories were reported to explain the issue, which depends on some theories as reported in previous literature.

The first theory is the embryonic nest cell theory which describes origin of squamous cell in thyroid gland from remnant of thyroglossal duct. The second is the metaplastic theory which suggests that it is due to chronic environmental stimulation that leads to metaplasia of the follicular epithelium. Lastly, dedifferentiation theory which was talking about 
existing (follicular, papillary, medullary, and anaplastic) thyroid carcinoma dedifferentiates into squamous cell carcinoma [5].

A few cases of Hashimoto's thyroiditis background with PSCC of the thyroid has been reported as in our case [6,8]. The association between Hashitmoto's thyroiditis and PSCC of the thyroid is controversial.

Management of thyroid PSCC mainly is complete surgical resection, the role of adjuvant therapy is unclear, due to lack of studies. Complete surgical resection was associated with a good survival rate in comparison with incomplete margin resection [7]. Radiation therapy and chemotherapy were not effective and do not affect the survival rate as reported in some literature [1].

Age at diagnosis, the extent of disease, and resection margins status were prognostic for overall survival. As reported, securing adequate resection margins was associated with better survival outcome [7].

Bone metastasis is the most common site [7], lung, liver and kidneys also found [1].

\section{Management of SCC}

\section{complete \\ surgical \\ resection}
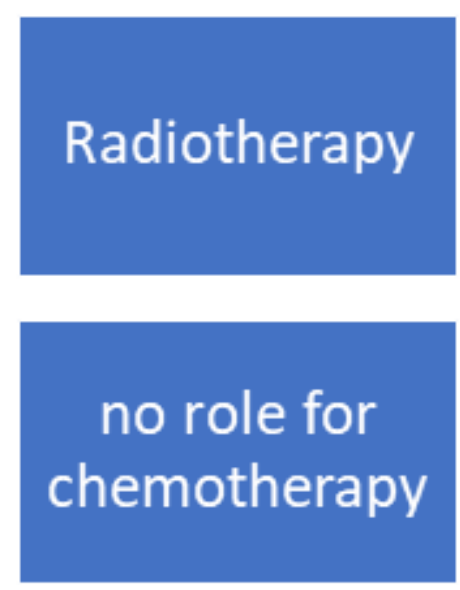

\section{Conclusion}

Radiotherapy is the main form of treatment of squamous cell carcinoma of the thyroid which may induce reduction in the size of the tumor and decrease pain. Radiotherapy can be given on neoadjuvant bases which may render resection possible in some patients. The tumor is usually not responsive to chemotherapy. The overall survival is poor regardless of the primary form of treatment, the median survival of the patients from the time of diagnosis is few months in most cases.

\section{References}

1. Au JK, Alonso J, Kuan EC, Arshi A, John MAS. (2017) Primary Squamous Cell Carcinoma of the Thyroid: A Population-Based Analysis.

2. Koyama S, Fujiwara K, Nosaka K, Fukuhara T, Morisaki T, et al. (2018) Immunohistochemical Features of Primary Pure Squamous Cell Carcinoma in the Thyroid. An Autopsy Case; 8504: 418-424.

3. Limberg J, Ullmann TM, Stefanova D, Finnerty BM. (2019). Prognostic Characteristics of Primary Squamous Cell Carcinoma of the Thyroid : A National Cancer Database Analysis. World J Surg. Springer International Publishing.

4. Saito K, Kuratomi Y, Yamamoto K, et al. (1981) Primary squamous cell carcinoma of the thyroid associated with marked leukocytosis and hypercalcemia. Cancer; 48: 2080-2083.

5. Chavan RN, Chikkala B, Biswas C, Biswas S, Sarkar DK. (2015) Primary Squamous Cell Carcinoma of Thyroid: A Rare Entity. Case Rep Pathol; 2015:1-3.

6. Chaudhary RK, Barnes EL, Myers EN. (1994) squamous cell carcinoma arising in hashimotos. Thyroiditis; 582-585.

7. Cho JK, Woo SH, Park J, Kim MJ, Jeong HS. (2014) Primary squamous cell carcinomas in the thyroid gland: An individual participant data meta-analysis. Cancer Med; 3(5): 1396-1403.

8. Shrestha M, Sridhara SK, Leo LJ, Iii GLC, Ehrhardt NM. (2018) Primary squamous cell carcinoma of the thyroid gland: A case report and review; $1-5$.

9. Othman RT, Baizeed AMA, Mohammed AA. (2020) Squamous cell carcinoma of the thyroid gland in an elderly female presenting as a rapidly enlarging thyroid mass. Int J Surg Case Rep; 70:119122. 